\title{
La sécurité nucléaire en 1983 : maturité et vigilance
}

\author{
Par Bernard AUGUSTIN \\ Secrétaire général du Comité interministériel de la sécurité nucléaire
}

L 'objectif de la sécurité nucléaire est simple dans son énoncé.

Assurer la protection des personnes et des biens contre les risques de toute nature que peut engendrer l'usage pacifique, sous toutes ses formes, de l'énergie nucléaire.

Les moyens d'atteindre cet objectif sont plus complexes: d'abord parce que le champ ouvert par l'énergie nucléaire en général est vaste et il l'est d'autant plus en France que notre pays s'est doté d'un programme électro-nucléaire important et complet. Elle inclut tout à la fois le parc des installations, l'extraction de l'uranium, son enrichissement, la fabrication des combustibles, leur retraitement, le stockage des déchets et des matières nucléaires et toutes les formes de transport.

Ensuite parce que, si l'utilisation de l'énergie nucléaire dans sa variété est l'affaire des exploitants, elle n'échappe pas à la vigilance des Pouvoirs publics, c'est-à-dire, à titres divers, de plusieurs départements ministériels.

Enfin parce que, plus que toute autre activité industrielle, l'utilisation de l'énergie sensibilise l'opinion publique et inquiète les populations quand elles sont mal informées.

Dès lors, les trois principes qui gouvernent l'organisation de la sécurité nucléaire dans notre pays s'imposent à l'évidence :

- une définition claire et précise des responsabilités de chacun : celle des exploitants comme celle des Pouvoirs publics, qu'il s'agisse des instances ministérielles ou des autorités départementales ;

- une coordination interministérielle assurant une cohérence des dispositions adoptées et multipliant l'efficacité de chacun des acteurs;
- une information complète et adaptée des populations et de leurs élus facilitant leur adhésion aux mesures de sécurité nucléaire, condition de leur bonne application pratique.

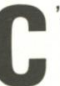
est dans l'esprit de ces principes qu'est intervenu en 1974 la création du Comité interministériel de la sécurité nucléaire et de son Secrétariat général, placé auprès du Premier Ministre, et permettant à ce dernier d'assurer la cohésion nécessaire et de prendre, si elle s'avérait nécessaire, la responsabilité personnelle des opérations face à une crise grave. Réunissant le Ministre de l'Intérieur et de la Décentralisation, le Ministre de I'Industrie et de la Recherche, le Ministre des Affaires sociales et de la Solidarité nationale, et le Ministre de la Défense, le Comité veille, à travers l'action de chacun de ses membres, à ce que soient réalisées les conditions de la sécurité nucléaire dans six grands domaines principaux :

- le contrôle technique de l'ensemble du programme nucléaire, contrôle qui constitue la sûreté nucléaire ;

- l'organisation des mesures et

l'action des Pouvoirs publics en cas d'accident ;

- la protection physique des installations ;

- la surveillance et la protection des matières nucléaires ;

- I'information ;

- le domaine international de la sécurité nucléaire.

Cette organisation qui existe depuis près de dix ans a acquis progressivement de la maturité et a permis de mettre en place dans chacun des domaines évoqués les dispositions techniques, réglementaires ou pratiques qui forment l'ensemble du dispositif de sécurité nucléaire.

Pendant cette période, une évolution se développait dans l'utilisation de l'énergie nucléaire. En effet, une longue période de plusieurs dizaines d'années a été nécessaire pour développer et promouvoir cette nouvelle source d'énergie. II fallait passer de la théorie à la pratique, c'est-à-dire, tout à la fois prendre les dimensions de l'industrialisation, répondre aux préoccupations légitimes de sûreté et insérer une nouvelle technologie, restée marquée par ses origines, dans une société mal préparée à la recevoir. Avec le temps, les installations en fonctionnement sont devenues de plus en plus nombreuses et progressivement les problèmes posés par l'exploitation succèdent à ceux posés par la conception et la réalisation.

$\mathrm{V}$ e sous l'angle de la sécurité nucléaire, cette évolution normale doit être prise en compte.

Premièrement, parce que plus le nombre des installations en fonctionnement est grand, plus les sources de risques sont nombreuses et plus doit être renforcée la vigilance. En ce sens, la création en 1979 d'une fonction d'inspection générale, auprès du Premier Ministre, répond au souci des Pouvoirs publics de s'assurer que l'ensemble des dispositions de sécurité nucléaire sont effectivement appliquées sur la totalité du territoire. En confiant cette mission au Secrétaire général du Comité interministériel de la sécurité nucléaire, le gouvernement a manifesté son souci d'éta- 
blir un lien direct entre la conception, la publication des mesures arrêtées au niveau national et la vérification pratique de leur application.

Deuxièmement, parce que si la construction d'une centrale nucléaire dure dix ans, son exploitation peut durer trente voire trentecinq ans. Cette nouvelle dimension de la durée a deux conséquences : elle permet d'accumuler l'expérience et, en ce sens, elle peut participer à l'amélioration des conditions de sécurité à condition que l'on veille à intégrer l'acquis de l'expérience dans le processus de recherche et de développement en faveur de la sécurité, processus qui a conditionné et conditionne encore l'utilisation de l'énergie nucléaire. Mais, seconde conséquence, elle conduit inexorablement à la routine. Si celleci résulte d'une maîtrise de plus en plus grande de la technique et des procédures, elle ne constitue pas, en soi, une menace pour la sûreté. Mais sérénité et bonne organisation ne doivent pas être synonyme d'immobilisme et l'expérience ne doit pas conduire à diminuer la vigilance.

Troisièmement, parce qu'au déroulement normal d'un programme nucléaire important correspondent la croissance du volume des matières nucléaires et le développement des déchets. Pour les premières, le stockage et le transport présentent des risques que la loi de 1980 a pris en compte en établissant une réglementation stricte sous la responsabilité du Ministre de I'Industrie et de la Recherche. En confiant au Secrétaire général du Comité interministériel de la sécurité nucléaire une mission dans le cadre de l'inspection du contrôle gouvernemental, le gouvernement a encore accentué la cohérence générale du dispositif de sécurité nucléaire.
Pour les seconds, une politique générale de gestion s'impose et met au premier rang les préoccupations de sécurité à court, moyen et long terme. En ce sens, une nouvelle étape a été franchie avec le débat énergétique au Parlement en octobre 1981 et, depuis, les travaux du Commissariat à l'Energie Atomique et les débats du Conseil supérieur de sûreté nucléaire sur le sujet on montré la nécessaire permanence de l'objectif de sécurité dans toute orientation visant la gestion des déchets radioactifs.

o uelles leçons doit-on tirer de cette évolution? Nous en retiendrons deux. Tout d'abord l'amélioration incessante des conditions de sûreté a considérablement réduit le nombre des incidents et des risques d'accidents significatifs. Du même coup, les enseignements pratiques issus de ces incidents, et encore plus, de ces accidents, sont relativement peu nombreux. Or le risque serait grand, pour les responsables de la sécurité nucléaire, de s'enfermer dans une certitude théorique peu compatible avec les réflexes indispensables face à une menace. L'ensemble des dispositifs prévus par les Pouvoirs publics touche un domaine très vaste qu concerne la radioprotection, la protection des populations, la communication des informations, etc L'accident de Three Mile Island a montré la difficulté de tout prévoir et surtout de tout appliquer à un moment imprévu. Si nous pouvons assurer aujourd'hui que l'ensemble de notre dispositif, amélioré par les leçons de Three Mile Island, est susceptible de répondre à une crise, nous n'en avons aucune assurance pratique.
A ce stade, la fiabilité et l'amélioration des dispositions prévues passent par la simulation. Une des meilleures manières de parvenir à une plus grande confiance dans notre système, à travers les organigrammes, les procédures, les plans, est la recherche d'une simulation adaptée, susceptible de poser le plus grand nombre de questions et de vérifier que nous sommes capables d'y répondre. Le complément de l'expérience, dans ce domaine, est l'exercice et le recours à l'imagination.

La seconde leçon que nous devons garder à l'esprit découle directement de la première. Que les spécialistes, les exploitants et les Pouvoirs publics aient confiance dans la sûreté des installations et dans les dispositifs de sécurité peut paraître légitime. Aujourd'hui, les faits leur donnent raison. Mais, du point de vue de la sécurité nucléaire en général, l'objectif ne serait qu'à moitié atteint si cette confiance n'est pas partagée par les populations et l'opinion publique en général. Toute entreprise qui vise à assurer les conditions de la sécurité sans en convaincre tous ceux qu'elle concerne risque de rater son but. N'oublions jamais que le bénéficiaire final de tout effort dans ce domaine est I'homme et que toute hypothèse de crise qui ne prendrait pas en compte l'adhésion et la nécessaire collaboration des populations est partielle et aléatoire. C'est dans ce sens que la réussite est indissociable du réalisme et du pragmatisme. La meilleure réglementation, le plan le plus élaboré ne sont efficaces que s'ils sont compris, connus et donc que si l'information a permis d'y associer toutes les personnes intéressées.

Ce n'est qu'à cette condition que l'énergie nucléaire, énergie sûre, aura sa pleine acceptation. 